\title{
Competitive conditioned reinforcement during differential reinforcement of low rates'
}

JOHN J. RANDOLPH AND WILLIAM R. SEWELL

UNIVERSITY OF ALABAMA

\section{Abstract}

Three pigeons were exposed to an FR-10 conditioned reinforcement probe superimposed over baselines generated by DRL schedules. Response rates increased when the probes were introduced.

\section{Problem}

Findley \& Brady (1965) have reported facilitative effects of response contingent magazine light presentations during large fixed ratio contingencies. In that study response rates were substantially increased by adding a concurrent FR-400 schedule of magazine light delivery to an FR-4000 food schedule.

The present study is the first in a series of attempts to demonstrate this effect when the high rates produced by the conditioned reinforcement contingency do not increase reinforcement density, without using programmed extinction as a baseline (Kelleher, 1961). Subjeets

The Ss were three adult silver king pigeons which were maintained at $80 \%$ of their ad. lib. body weight. They had extensive histories, i.e., several hundred hours, of both DRL-20 sec. and DRL-30 sec. schedules of food reinforcement.

\section{Apparatus}

A standard one cubic foot experimental chamber was used. Illumination of the entire chamber was provided by a 7-w lamp located opposite the response panel near the ceiling. The translucent plastic response key, 8-3/4 in from the floor on the midline of the response panel, was illuminated by a green $7-w$ lamp. The aperture to the grain magazine was centered 2 in from the floor immediately under the key. Programming was accomplished with standard relay equipment.

\section{Procedure}

The Ss were exposed to a DRL-20 sec. schedule of food reinforcement until their intrasession rates of responding were relatively consistent, and the intersession variability was small. The DRL schedule specifies a minimum interresponse time, or pause length, and in this experiment each response was reinforced whose latency was equal to or exceeded the specified value. Reinforcement was a 4.5 sec. access to grain.

A probe (conc. DRL-20 sec. FR-10) was introduced within several sessions. The DRL contingency was unchanged by the probe, and every tenth response produced a 2 sec. blackout of the box during which the magazine light was illuminated. These stimulus conditions were identical to those which occurred at food reinforcement on the DRL schedule except the grain hopper was not elevated.

Two of the three birds were re-exposed to a DRL-30 sec. schedule in the latter portion of the experiment. The probe, now conc. DRL-30 sec. FR-10, was reintroduced when the intrasession variability was small enough for an adequate evaluation of the effects of the fixed ratio contingency.

\section{Results and Diseussion}

Increases in response rates during the probes of the $20 \mathrm{sec}$. baseline were found for all three birds, but in two of the three cases the changes were small and transitory. The most dramatic change is shown in the first probe in Fig. 1. The segment marked with the letter A shows a food reinforcement delivered while the probe was in effect.

The amount of rate change during the probes was inversely related to the adequacy of each bird's performance on the DRL schedule, e.g., the bird with the lowest rates on the DRL schedule showed the least increase in rate during the probe. This correlation suggested that the strength of the effect might be in part a function of several variables which are inextricably confounded by the DRL schedule. These are reinforcement density, local response rates, and overall response rates.

The two birds which showed the least rate change during the probes of the 20 sec. baseline were shifted to a DRL-30 sec. schedule. Previous work in this laboratory has indicated that this shift effects a slight rise in overall response rate with a resultant decrease

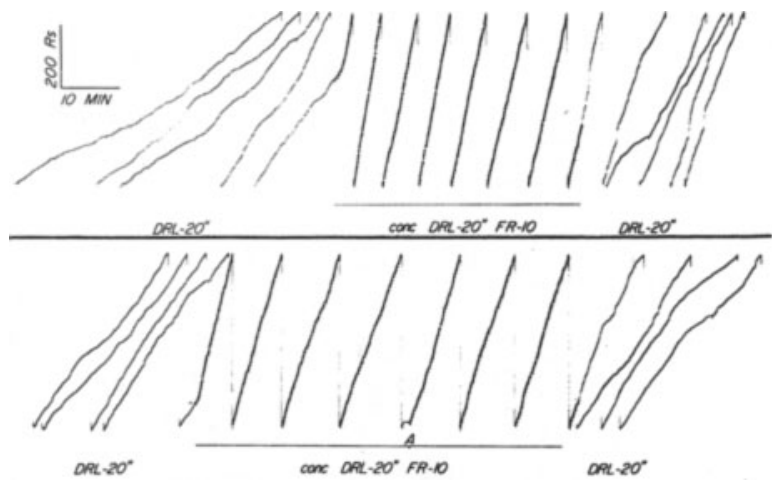

Fig. 1. The cumulative record of an entire session for SK-1. The response pen is deflected during food reinforcement on the DRL-20" schedule. During conc. DRL-20" FR-10, magazine light presentations are indicated by a deflection of the response pen, and food reinforcements are indicated on the event pen. Part of the record has been collapsed, and no omitted portions of the event record contained reinforcement signals. Arrows mark the first and last magazine light presentations for each probe. 


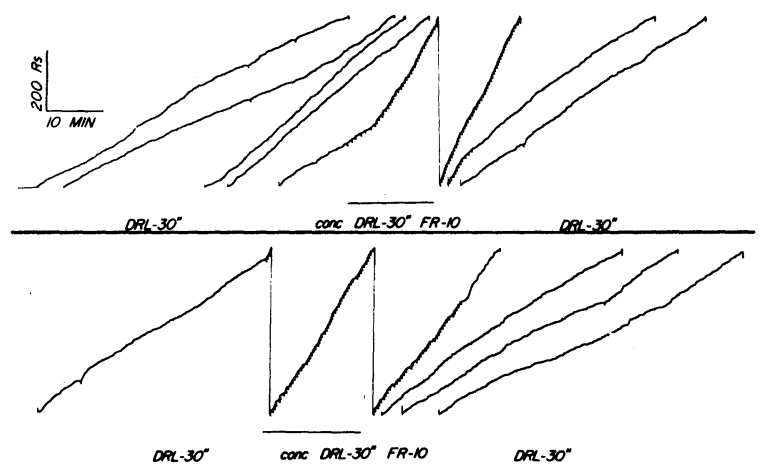

Fig. 2. The cumulative record for an entire session for SK-2. The recording specifications are identical to those for Fig. 1.

in reinforcement density (Randolph \& Sewell, 1965). The DRL-30 sec. baseline performances of these two birds was similar to the DRL-20 sec. baseline performance shown in Fig. 1. Also, the effect of the fixed ratio probe was greater for these two birds during the DRL-30 sec. baseline than it had been during the DRL-20 sec. baseline. An example of the effects of probes on a DRL-30 sec. baseline is given in Fig. 2.

An examination of the cumulative records produced by all three Ss during post experimental exposure to DRL-20 sec. revealed frequent extended bursts of responding at rates higher than had been observed prior to their experience with the probes. A history of exposure to fixed ratio schedules has been reported to have a similar effect on DRL performance (Sidman, 1961, p. 312).

These results tentatively extend the generality of the findings of Findley \& Brady (1965) to a situation in which the effects of the conditioned reinforcement paradigm oppose the requirements of the baseline schedule. However, these data also suggest that the generality of the effects of the concurrent DRL-X, FR-10 contingency may be limited. It is well known that the terminal performance of pigeons on DRL schedules is less efficient than in other animals, unless special training procedures are employed (Staddon, 1965). The two pigeons in this study who showed small and transitory effects of the conditioned reinforcement paradigm had DRL-20 sec. baseline performances which more closely approximated performances considered normal for other species than did the pigeon whose data appears in Fig. 1. Conversely, these same two birds were quite sensitive to the FR contingency when their baseline behavior was less efficient, i.e., during DRL-30 sec. baseline sessions. It seems possible, therefore, that some factor related to the amount of control exerted by the DRL schedule was the relevant variable which determined the effect of the FR-10 contingency.

Additional research is currently in progress to ascertain the specific properties of the baseline performance which determines its sensitivity to the fixed ratio contingency. Research with rats is also in progress in an attempt to replicate the main effects described above.

\section{Referenees}

Findley, L. D., \& Brady, J. V. Facilitation of large ratio performance by the use of conditioned reinforcement. J. exp. Anal. Behav., 1965, 8, 125-130.

Kelleher, $\mathbf{R}$. T. Schedules of conditioned reinforcement during experimental extinction. J. exp. Anal. Behav., 1961, 4, 1-5.

Randolph, J. J., \& Sewell, W. R. An analysis of "bursts" during DRL performance in the pigeon. Presented at the meeting of the Southeastern Psychological Ass., Atlanta, April, 1965.

Sidman, M. Tactics of Scientific Research. New York: Basic Books, Inc., 1961.

Staddon, J. E. R. Some properties of spaced responding in pigeon. J. exp. Anal. Behav., 1965, 8, 19-30.

Note

1. This research was supported by National Institute of Health predoctoral research fellowships 5 FI1 MH15 746-02 and 1 F1 MH-24 644-01 to the first and second authors respectively. 